UDC: 330.34:339.977[330.47+336.742]

DOI: 10.26697/ijes.2019.2.41

\section{Genesis of IT Economy: from Cryptocurrency to Digital Asset}

Associate Professor Pypenko I. S. ${ }^{1,2}$, Kud A. A..$^{3,4}$

${ }^{1}$ Kharkiv Regional Public Organization "Culture of

Health" (KRPOCH), Ukraine

${ }^{2}$ Scientific Research Institute KRPOCH, Ukraine

${ }^{3}$ Simcord Information Services SIA, Latvia

${ }^{4}$ Simcord LLC. Technologies, Ukraine

\begin{abstract}
Background:

Globalisation of the world economy and computarisation of financial systems form a new branch - IT economy. There appear some alternative currencies generated by computarisation of financial systems. A remarkable example of this process is cryptocurrencies which have currently taken up financial markets, stock-markets and exchange platforms. Nowadays a cryptocurrency is accepted by many American, European and Asian companies as a payment means. However the legal and regulatory framework, in particular of Ukraine, has not been arranged yet. There are no legal bases, instructions, or clarifications as for the cryptocurrencies market. It is resulted in currency risks among subjects in the sphere of conducting accounts and payments. So, the problem of using cryptocurrencies and ascertaining tendencies of their development and functioning has appeared to be extremely urgent.
\end{abstract}

The aim of the research is to study genesis of IT economy, determine the current state and perspectives of cryptocurrencies and digital assets functioning.

\section{Methods:}

Methodology of the research is grounded on the system approach to studying formation history and a prognostic aspect of IT economy development. A complex of theoretical research methods has been used: deduction and induction, analysis and synthesis, comparison, generalisation, systematisation and interpretation of results.

\section{Results:}

The history of IT economy formation has been researched, in particular the usage of cryptocurrencies. The system of DigiCash (David Chaum) started using cryptography in 1990 to provide confidentiality of accounts and payments. Still, the term "cryptocurrency" has been used only since 2009, when Bitcoin peer-to-peer payment system appeared, due to Satoshi Nakamoto. Hashing SHA-256 and the proofof-work system have been used.

For over ten years of the cryptocurrency market in existence, more than two thousand of its types have appeared. The following types of cryptocurrencies can be outlined among the most widespread: Bitcoin, Ethereum, XRP, Bitcoin Cash, Litecoin, NEO, Binance Coin, Tether, Bitcoin SV, Stellar, TRON, Cardano, Monero etc.

General market capitalisation of all types of cryptocurrencies increases every year. It reached its peak at $\$ 813.8$ milliard on the date of 7.01.2018. After that there were significant fluctuations and index decrease to \$ 102.0 milliard (14.12.2018). In 2019 the index gradually increased to $\$ 274.1$ milliard (30.05.2019). It should be noted that the market capitalisation of a cryptocurrency cannot be considered an appropriate means of measuring and ranking a currency value and importance. This index does not determine the amount of capital invested into the currency and the quality of a cryptocurrency.

Digital coins have been used as money: goods are sold and services are provided with their help, they can be saved as well. In countries with developed economy bitcoins are considered to be: as possession and exchange commodity in the USA, a payment means in Japan, a virtual currency and a payment means in European Union (with amendments in national laws). Bitcoins are banned in such countries as Bangladesh, Bolivia and Ecuador. Trading on the crypto-markets is restricted in China.

The company Simcord is one of the leaders in the sphere of IT economy. The empirical experience has allowed developing conceptual and categorical framework in the sphere of IT economy; forecasting prospects of this branch development in direction form a cryptocurrency to a digital asset which has advantages over it; initiating regulatory and legal support of a digital asset at the country level.

\section{Conclusions:}

Despite the local bans and restrictions, a cryptocurrency has its own growth potential. However a digital asset is more functional and reliable. It is presented by an informational resource, displayed by a code, can be circulated both in a distributed register and other fields. It determines the right for usage, levels mediators' services, etc.

There are a lot of unsolved issues concerning methodological, technological, legal and other aspects of the IT economy sphere: what alternative technologies will provide efficiency of cryptocurrencies and digital assets functioning; whether it is worth issuing electronic forms of national currencies and/or cryptocurrencies and blockchains.

\section{Information about the authors:}

Pypenko Iryna Sergiivna - Doctor of Philosophy in Economics, Associate Professor, Kharkiv Regional Public Organization "Culture of Health", Scientific Research Institute KRPOCH, Kharkiv, Ukraine.

Research interests: theoretical and methodological problems of economics, digital asset management; https://orcid.org/0000-0001-5083-540X.

Kud Aleksandr Aleksandrovich - Founder and Director of Simcord Information Services SIA, Riga, Latvia; Founder and Director General of Simcord LLC. Technologies, Kharkiv, Ukraine.

Research interests: digital asset, IT economy, capital management, Bitbon System development; https://orcid.org/0000-0001-5753-7421.

\section{Corresponding Author:}

Pypenko Iryna Sergiivna

Corresponding Author's Email:

edu.center.office@gmail.com 